\title{
Descomposición sectorial de la productividad total de los factores en Chile, 1996-2010
}

\author{
Patricio Aroca ${ }^{2}$ y Nicolás Garrido 3
}

\section{Resumen}

En este artículo se descompone la productividad total de los factores (PTF) por sectores económicos utilizando los datos contenidos en las matrices de insumo-producto de Chile de los años 1996, 2003 y 2010. El análisis se enfoca en tres efectos: el efecto del cambio en la demanda sectorial; el efecto del cambio en la receta de producción y las transacciones intersectoriales, y el efecto del cambio tecnológico sectorial. Este análisis permitió observar que los dos últimos son los principales factores que explican las variaciones de la productividad en el período considerado. En este contexto, destacan los siguientes sectores: industria manufacturera; electricidad, gas y agua, e intermediación financiera y servicios empresariales. Estos han encabezado los cambios positivos de la productividad del país, mientras que el sector de servicios personales podría estar actuando como un factor limitante de su crecimiento. En la descripción del estudio se matizan y contextualizan estas conclusiones.

Palabras clave

Productividad, medición, análisis de insumo-producto, análisis de factores, Chile

Clasificación JEL

O41, O47, C67

Autores

Patricio Aroca es Profesor Titular de la Escuela de Negocios de la Universidad Adolfo Ibáñez, Chile. patricio.aroca@uai.cl

Nicolás Garrido es Profesor Asociado del Departamento de Economía de la Universidad Diego Portales, Chile. nicolas.garrido@udp.cl

1 Los autores agradecen los comentarios de los asistentes a las presentaciones de versiones preliminares de este artículo en seminarios que tuvieron lugar en la Universidad Adolfo Ibáñez y en seminarios del Núcleo Intelis, Iniciativa Científica Milenio.

2 El autor agradece el financiamiento recibido del Fondo de Fomento al Desarrollo Científico y Tecnológico (FONDEF), en el marco del proyecto "Medidor de impacto y derrames de proyectos regionales en Chile" (código CA13110061).

3 El autor agradece el financiamiento recibido del Fondo Nacional de Desarrollo Científico y Tecnológico (FONDECYT), en el marco del proyecto "Análisis de la productividad regional y sectorial y su influencia en la economía agregada" (№ 1140131). 


\section{Introducción}

Hay dos aseveraciones que destacan en los estudios de Prescott (1998) y Easterly y Levine (2002). Según la primera, de carácter empírico, el mejor constructo que permite diferenciar las tasas de crecimiento y el producto interno bruto (PIB) de distintos países es la productividad total de los factores (PTF) (Solow, 1957). De acuerdo con la segunda, de carácter teórico, no hay consenso sobre el concepto de PTF, lo que impide definirla y medirla sin ambigüedad. Estas dos afirmaciones han dado origen a numerosos debates e investigaciones en los medios académicos. El presente estudio contribuye a esos debates, mediante la propuesta de una nueva forma de medición de la PTF para el caso de Chile, que supone dos innovaciones respecto de los cálculos anteriores. Específicamente, se mide la PTF mediante matrices de insumo-producto y se descompone la PTF de toda la economía, conforme a los aportes de 12 sectores económicos.

El estudio de la PTF de Chile abarca diferentes períodos y, dependiendo de la información existente, se centra en los niveles macro, meso y microeconómico. La mayor disponibilidad de información agregada permite realizar más investigaciones sobre la PTF en la macroeconomía. Fuentes, Larraín y Schmidt-Hebbel (2006) demuestran que el aumento de la productividad en los períodos de alto y moderado crecimiento (1990-1997 y 1998-2005, respectivamente) dio origen a más del $60 \%$ del crecimiento económico ${ }^{4}$. Estos autores consideran que la PTF se relaciona con componentes estructurales y cíclicos de la economía. Mientras que en períodos previos a los años noventa los factores estructurales, entre otros la estabilidad macroeconómica, desempeñaron un rol importante, esta situación se revirtió en los últimos 20 años, etapa en la que las fluctuaciones de la PTF obedecieron fundamentalmente a efectos cíclicos como los términos de intercambio (el precio del cobre) y la devaluación del tipo real de cambio. Chumacero y Fuentes (2006) sostienen que un modelo de crecimiento exógeno es el mejor instrumento para interpretar los datos económicos, en los que se observan una tendencia estacionaria de la PTF e incrementos temporales derivados del perfeccionamiento de la calidad del capital, calculado mediante la comparación de los precios de los bienes de capital y los de consumo; una mejora de los términos de intercambio, y una reducción de las distorsiones de las medidas oficiales, entre otras el gasto del gobierno. Los autores señalan que cuando dichos incrementos se deben a las políticas vigentes, el cambio puede ser de largo plazo, en tanto que los cambios transitorios dan origen a fluctuaciones de la productividad.

Progresivamente, a medida que las mejoras en materia de productividad derivadas de la estabilidad macroeconómica comenzaban a agotarse, se empezó a estudiar la influencia que ejercía en la PTF la microeconomía y su complementación con la estabilidad institucional. Así, sobre la base de los abundantes datos provenientes de la Encuesta Nacional Industrial Anual (ENIA), se realizaron estudios en los que se establecieron vínculos entre las empresas y la productividad. Pavcnik (2002) encontró evidencias de que la apertura comercial había elevado la productividad de las empresas. Asimismo, se formularon varias hipótesis sobre los canales de transmisión de las mejoras, según las cuales serían los siguientes: i) una mayor especialización; ii) la expansión de los mercados potenciales, con el fin de aprovechar las economías de escala; iii) la difusión de innovaciones tecnológicas y de buenas prácticas de administración, y iv) la reducción de las prácticas anticompetitivas que pudieran darse a nivel local. Bergoeing, Hernando y Repetto (2005) señalan que el aumento de la PTF de las empresas obedeció a mejoras introducidas en estas, no a la reubicación de factores que podría haber sido inducida por un proceso schumpeteriano de "muerte y nacimiento" de firmas. La estructura del mercado del trabajo y su efecto en la productividad fueron estudiados por Álvarez y Fuentes (2009), quienes señalan que el alza de los salarios mínimos puede haber contribuido a la reducción de la PTF

\footnotetext{
4 Esta conclusión es obtenida de acuerdo con el método empleado por Fuentes, Larraín y Schmidt-Hebbel (2006), según el cual la medida de la PTF se estima a partir de un acervo de capital que se calcula ajustando por el consumo de energía y el empleo mediante la tasa de salario y de las horas trabajadas. Los resultados no son muy diferentes usando las otras medidas.
} 
en los últimos años y afectado principalmente a las empresas que tienen una mayor proporción de trabajadores poco capacitados.

La mesoeconomía ${ }^{5}$ del país y su relación con la productividad son temas que recibieron una atención más tardía, específicamente en los estudios de Álvarez y Fuentes (2004), Vergara y Rivero (2006) y Fuentes (2011). Este último autor muestra que, hacia fines de los años noventa, se produjo un quiebre estructural en la productividad laboral de muchos sectores (agricultura, pesca y silvicultura; minería; comercio, restaurantes y hoteles, y transporte y comunicaciones), y que este parece estar relacionado con el efecto de los cambios estructurales y los componentes cíclicos en la productividad macroeconómica (Fuentes, Larraín y Schmidt-Hebbel, 2006). La descomposición del incremento de la productividad del empleo en dos categorías - sectorial y atribuible a la reasignación de empleo entre sectores - permitió a los autores determinar que más del 97\% del aumento correspondió a la categoría sectorial y solo el 3\% a la referida a la reasignación. Los sectores de bienes transables (minería e industria manufacturera) registran una profundización del uso de capital que actuó como motor del aumento de la productividad laboral, mientras que la PTF incidió significativamente en la productividad laboral de los sectores de comercio, restaurantes y hoteles, y transporte y comunicaciones. Álvarez y Fuentes (2004) llegan a una conclusión similar: que la expansión de los sectores de minería; manufactura, y agricultura, silvicultura y pesca en los años noventa fue consecuencia de la acumulación de capital y de la PTF, mientras que la del sector de bienes no transables en la misma década obedeció fundamentalmente a la acumulación de capital. Vergara y Rivero (2006) dan a conocer conclusiones que ponen en evidencia el heterogéneo desempeño de los sectores de bienes transables. Dos de estos (agricultura, silvicultura y pesca, y minería) presentan tasas de crecimiento de la PTF superiores a la de acumulación de capital durante el período 19962001 y solo en uno (industria manufacturera) se observa la situación inversa. Según Fuentes (2011), los sectores de construcción y servicios financieros podrían haber tenido una tasa de crecimiento de la PTF muy baja ${ }^{6}$, mientras que Vergara y Rivero (2006) afirman que el último de estos ocupa el segundo lugar en lo que respecta al incremento de la PTF en el período 1986-2001.

Esta gran diversidad de resultados nos invita a realizar una triple reflexión. En primer lugar, los criterios técnicos y las fuentes de datos empleados para medir la productividad del país son muy variados, aun cuando la base conceptual de las mediciones siga siendo la descomposición de la contabilidad del crecimiento. En segundo término, la hipótesis de Harberger (1998), según la cual el incremento de la productividad se concibe como hongos y las reducciones de costos pueden deberse a "1.001 posibles causas" en diferentes sectores, cuyo aumento de la productividad adopta muy distintas formas, parece aplicarse al caso chileno. En tercer lugar, la heterogeneidad de resultados también nos invita a establecer una relación entre la productividad microeconómica, mesoeconómica y macroeconómica, para posibilitar una lectura integrada y desde una perspectiva amplia de las diferentes conclusiones.

En este artículo se dan a conocer dos nuevas modalidades de análisis de la PTF sectorial en Chile. En primer término, organizamos la información proporcionada por el Banco Central en las matrices de insumo-producto de los años 1996, 2003 y 2010 y la compatibilizamos con los datos pertinentes del Instituto Nacional de Estadísticas. A continuación, realizamos una descomposición de la PTF total de la economía en función de las contribuciones sectoriales, los efectos de la demanda y el efecto de la interacción entre los sectores. La comparación de la productividad intersectorial se enmarca en el análisis de la contabilidad del crecimiento de Solow y las observaciones de Baumol y Wolff (1984).

5 Usamos el concepto de mesoeconomía para describir entidades claramente identificables en la economía como los sectores económicos y las regiones de un país, pero que no representan directamente el comportamiento microeconómico de compra y venta en mercados, ni describen propiedades agregadas de toda la macroeconomía.

6 Este supuesto es tentativo, dado que se basa en la conclusión de Fuentes (2011), de acuerdo con la cual se dio un proceso de intensificación del uso del capital unido a un crecimiento prácticamente nulo de la productividad laboral. 
Los resultados de esta investigación permiten construir una taxonomía de los sectores, conforme a su capacidad para dar impulso a la economía y las variaciones de la productividad sectorial. En esta taxonomía sobresalen los sectores de industria manufacturera; electricidad, gas y agua, e intermediación financiera y servicios empresariales, que encabezaron el aumento de la PTF en el período 1996-2010. Estos sectores registraron un incremento de la productividad, que difundieron a todo el tejido productivo.

En este contexto, el sector de servicios personales podría estar limitando la expansión de la productividad de la economía, ya que mostró una reducción de esta en el período considerado, lo que tuvo un gran impacto en todo el sistema productivo.

Aparte de la Introducción, este artículo se divide en tres secciones. En la sección II se describe el método de descomposición empleado para analizar la productividad. En la III se explica el origen de los datos y se analizan los resultados de la descomposición. En la IV se presentan las conclusiones. El artículo concluye con un anexo en el que se detallan los métodos empleados para realizar los cálculos.

\section{Método de descomposición}

La PTF indica los aumentos de la producción que no se deben a un incremento de los factores de producción primarios, es decir, del capital y el trabajo. Las técnicas de medición empleadas son múltiples, y existe una amplia literatura sobre la materia sintetizada en varios compendios como los de Del Gatto, Liberto y Petraglia (2011), y Timmer y otros (2010).

Para la descomposición y medición de la PTF, se utilizó la información contenida en las matrices de insumo-producto de Chile. De acuerdo con la clasificación de las principales mediciones de productividad elaborada por la Organización para la Cooperación y el Desarrollo Económicos (OCDE, 2001), el procedimiento empleado se puede definir como multifactorial. La medición aplicada en el estudio se distingue de la referida a la productividad del capital $(K)$, el trabajo $(L)$, la energía $(E)$, los materiales (M) y los servicios (S), definida en el manual de la OCDE como KLEMS, para la que se considera el producto bruto, mientras que en este caso se emplea el valor agregado. Como se indica en el cuadro 3 del manual de la OCDE (2001), la diferencia entre ambas mediciones es importante. Sin embargo, y con el fin de posibilitar la comparación de los resultados expuestos en este artículo y los presentados en otros estudios sobre la productividad en Chile, se optó por hacer los cálculos sobre la base del valor agregado.

El empleo de las matrices de insumo-producto permite calcular una medida de la productividad que puede descomponerse por aportes sectoriales conforme a un marco analítico coherente, como se señala en el manual mencionado de la OCDE. Para la descomposición sectorial de la PTF se recurre al método aplicado por Wolff (1984 y 1985), con el propósito de describir la composición de los cambios productivos que influyeron en la reducción del crecimiento de la productividad de los Estados Unidos después de la Segunda Guerra Mundial.

De conformidad con el modelo formal, se definieron las siguientes variables:

$x_{t}$ : vector columna del producto bruto sectorial en el período $t$.

$y_{t}$ : vector columna de la demanda final de cada sector en el período $\mathrm{t}$.

$A_{t}$ : matriz de coeficientes intersectoriales en el período t.

$l_{t}$ : vector fila de los coeficientes de trabajo en el período t, que indica el volumen de empleo necesario en cada unidad de producción.

$k_{t}$ : vector fila de los coeficientes de capital en el período t, que indica la cantidad de capital requerido en cada unidad de producción. 
$p_{t}$ : vector fila de los precios en el período t, que indica el precio de cada unidad producida en las diferentes industrias.

Además, se definieron los siguientes escalares:

$w_{t}$ : tasa del salario promedio de todos los sectores en el período $t$.

$r_{t}$ : tasa de beneficio del acervo (stock) de capital en el período t.

$Y_{t}=p_{t} y_{t}$ : producto nacional bruto a precios corrientes del período $\mathrm{t}$.

$L_{t}=l_{t} x_{t}$ : empleo total en la economía en el período t.

$K t=k_{t} x_{t}$ : acervo total de capital en la economía en el período t.

La aplicación de la técnica de contabilidad del crecimiento de Solow (1957), para la que solo se requieren los supuestos de rendimientos constantes a escala y un entorno de competencia perfecta, permite definir la productividad total de los factores en los siguientes términos:

$$
\rho=\frac{d Y}{Y}-\frac{w L}{Y} \frac{d L}{L}-\frac{r K}{Y} \frac{d K}{K}
$$

Si se considera la información derivada del modelo de insumo-producto, los valores se pueden expresar de la siguiente manera:

$$
\rho=\frac{(p d y-w d L-r d K)}{Y}
$$

Dado que es posible expresar la demanda sectorial como la diferencia entre la producción bruta y las compras intermedias,

$$
y=(I-A) x
$$

Por consiguiente, se realiza la siguiente distinción:

$$
d y=(I-A) d x-(d A) x
$$

De igual manera, se pueden diferenciar el empleo y el capital como se indica a continuación:

$$
\begin{gathered}
d L=l d x+(d l) x \\
d K=k d x+(d k) x
\end{gathered}
$$

Si se realizan las sustituciones pertinentes en la ecuación (1),

$$
\rho=\frac{(p(I-A) d x-p(d A) x-w l d x-w(d l) x-r k d x-r(d k) x)}{Y}
$$

Para simplificar esta expresión, se emplea la identidad según la cual el precio de un bien cubre el costo de los bienes intermedios y el pago de los factores. En términos formales,

$$
p=p A+w l+r k
$$

O

$$
p(I-A)=w l+r k
$$


Si se realizan las sustituciones pertinentes en la ecuación (2), se obtiene el siguiente resultado:

$$
\rho=\frac{-(p d A+w d l+r d k) x}{Y}
$$

En el estudio de Miller y Blair (2009), se define la productividad de un sector, denominado $j$, de la siguiente manera:

$$
\pi_{j} \equiv \frac{-\left(p d a_{. j}+w d l_{j}+r d k_{j}\right)}{p_{j}}
$$

donde $a_{. j}$ corresponde a la columna $j$ de la matriz $A$ y representa el número de unidades necesarias desde el sector intermedio $i$ para producir en el sector $j$. Por su parte, $l_{j}$ y $k_{j}$ representan el número de unidades de trabajo y capital, respectivamente, necesarias para producir una unidad del sector $j$. Por lo tanto, la productividad del sector $j$ aumenta cuando se emplean menos insumos y factores para la producción de una unidad a precios constantes.

Con esta definición es posible expresar la productividad agregada de la ecuación (3) en estos términos:

$$
\frac{\pi \hat{p} x}{Y}
$$

donde $\pi$ es el vector columna de la productividad sectorial y $\hat{p}$ es el vector de precios diagonalizado.

A continuación, aplicando la relación $x=(I-A)^{-1} y$ y la identidad $I=\hat{p}^{-1} \hat{p}$, es posible expresar la productividad agregada como

$$
\rho=\frac{\pi \hat{p}(I-A)^{-1} \hat{p}^{-1} \hat{p} y}{Y}
$$

Para simplificar la notación, la ecuación anterior se puede expresar de la siguiente manera:

$$
\rho=\pi S \beta
$$

donde $\pi$ es un vector fila, en el que cada componente $j$ representa la productividad del sector correspondiente. La matriz $S=\hat{p}(I-A)^{-1} \hat{p}^{-1}$ ilustra los efectos económicos de las operaciones entre las industrias. Cada término $s_{i, j}$ de la matriz $S$ puede expresarse como $s_{i, j}=\frac{l_{i, j} p_{i}}{p_{j}}$, donde el multiplicador técnico ${ }^{7}, l_{i, j}$ representa el aumento directo e indirecto generado en la producción física del bien $i$ como consecuencia de incrementar la demanda del bien $j$. Por consiguiente, $s_{i, j}$ representa el multiplicador del bien $i$, derivado del acrecentamiento de su demanda, en comparación con el precio del bien $j$. Lo anterior podría resumirse diciendo que, si en lugar de tomar en cuenta el multiplicador $l_{i, j}$, que incluye los efectos directos e indirectos, se considerara solo el efecto directo del coeficiente técnico $a_{i, j}$ de la matriz de coeficientes técnicos $A$, los efectos económicos se expresarían como $\tilde{s}_{i, j}=\frac{a_{i, j} p_{i}}{p_{j}}$, donde $\tilde{s}_{i, j}$ representa el costo del insumo $i$ comparado con el del bien $j$.

Finalmente, $\beta=\frac{\hat{p} y}{Y}$ es un vector fila, en el que cada posición $j$ representa la participación del sector $j$ en la demanda final de la economía. Este vector también puede interpretarse como la preferencia de gasto en determinados sectores que muestra la demanda del país.

Si se considera nuevamente la descomposición expresada en la ecuación (4) y se ignoran los efectos de segundo orden, es posible aproximar linealmente el cambio en la PTF de la siguiente manera:

7 En términos de la notación estándar de Leontief, $L=(I-A)^{-1}$, por lo que $l_{i, j} \in L$. 


$$
\Delta \rho=\pi S \Delta \beta+\pi \Delta S \beta+\Delta \pi S \beta
$$

donde el primer término de la derecha representa el cambio en la productividad derivado del cambio en las preferencias de la demanda, el segundo alude al cambio atribuible a los efectos intersectoriales y el tercero representa los cambios intrasectoriales.

\section{Datos y resultados}

Para realizar los cálculos descritos en este artículo, se utilizó información proveniente de las bases de datos de las cuentas nacionales del Banco Central de Chile correspondiente a los años 1996, 2003 y 2010. También se empleó información del Instituto Nacional de Estadísticas, para calcular el salario promedio por hora de los trabajadores en cada año, y se consideraron los datos sobre acervo de capital sectorial presentados por Henríquez (2008).

Fuentes, Larraín y Schmidt-Hebbel (2006) ofrecen evidencias de que el comportamiento de la PTF se vincula a los componentes cíclicos de la economía. Así, para ofrecer un marco de referencia analítico, en el gráfico 1 se ilustra el componente cíclico, calculado mediante la aplicación del filtro de Hodrick y Prescott (1981) $(\lambda=1.600)$ del producto interno bruto a precios constantes. Asimismo, en las barras verticales (años 1996, 2003 y 2010) se presentan los datos de insumo-producto empleados en el cálculo.

Desde 1996 hasta 2003, la economía creció a una tasa anual aproximada del 3,5\% y se observa que esta expansión se dio en el contexto de una fase contractiva. En el siguiente período, que se extiende desde 2003 a 2010, el crecimiento de la economía ascendió a un 4,5\%.

Gráfico 1

Ciclo económico, 1986-2010

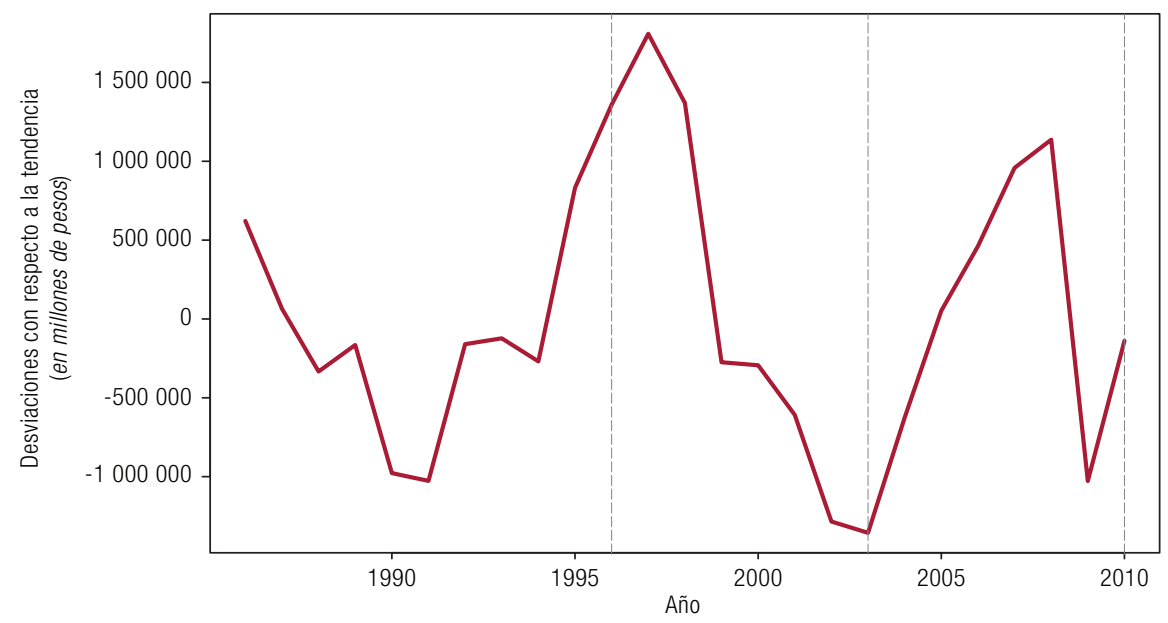

Fuente: Elaboración propia, sobre la base de datos del Banco Central de Chile.

En el cuadro 1 se presenta el incremento del valor bruto de producción (VBP) de los sectores en los dos períodos considerados, en los que tres de ellos registraron tasas anuales medias superiores al 5\%: minería; electricidad, gas y agua, e intermediación financiera y servicios empresariales. En un segundo grupo (administración pública, servicios personales, y comercio, hoteles y restaurantes) las tasas de crecimiento fueron moderadas, en tanto que en un tercero (pesca, transporte y comunicaciones, e industria manufacturera) se produjo una considerable desaceleración. 
Cuadro 1

Incremento anual del valor bruto de la producción (VBP)

(En porcentajes)

\begin{tabular}{lcc}
\hline Sector & $1996-2003$ & $2003-2010$ \\
\hline Agropecuario-silvícola & 3,2 & 1,6 \\
\hline Pesca & 5,5 & $-2,8$ \\
\hline Minería & 7,2 & 11 \\
\hline Industria manufacturera & 3,8 & 0,9 \\
\hline Electricidad, gas y agua & 5,3 & 9,2 \\
\hline Construcción & 0,4 & 3,9 \\
\hline Comercio, hoteles y restaurantes & 3,3 & 3,6 \\
\hline Transporte y comunicaciones & 8,9 & 1 \\
\hline Intermediación financiera y servicios empresariales & 7,4 & 6,1 \\
\hline Servicios de vivienda & 1,1 & 2,5 \\
\hline Servicios personales & 5,6 & 3,8 \\
\hline Administración pública & 3,5 & 4,6 \\
\hline Todos los sectores & 4,51 & 3,68 \\
\hline
\end{tabular}

Fuente: Elaboración propia.

\section{Salario y coeficientes técnicos del trabajo}

Para calcular el salario promedio, se utilizó información del Instituto Nacional de Estadísticas sobre la distribución del empleo en los 12 sectores en 1996, 2003 y 2010 (véase el cuadro 2). Esta información se complementó con datos del Banco Central de Chile correspondientes a los mismos años, según los cuales el número de horas semanales trabajadas fue aproximadamente 46, 43,3 y 40, en promedio y respectivamente. La multiplicación de estas cifras por el número de integrantes de cada sector permitió calcular el vector $l_{n}$, que indica el total de horas trabajadas por sector.

Cuadro 2

Número de trabajadores por sector

(En miles)

\begin{tabular}{lrrr}
\hline Sector & 1996 & 2003 & 2010 \\
\hline Agropecuario-silvícola & 723,17 & 710,96 & 703,24 \\
\hline Pesca & 66,17 & 81,3 & 45,62 \\
\hline Minería & 92,37 & 79,33 & 199,17 \\
\hline Industria manufacturera & 844,89 & 822,73 & 799,31 \\
\hline Electricidad, gas y agua & 39,22 & 26,2 & 58,88 \\
\hline Construcción & 406,21 & 461,7 & 562,5 \\
\hline Comercio, hoteles y restaurantes & 925,96 & 1129,12 & 1746,53 \\
\hline Transporte y comunicaciones & 387,21 & 484,38 & 522,57 \\
\hline Intermediación financiera y servicios empresariales & 353,08 & 471,12 & 574,64 \\
\hline Servicios de vivienda ${ }^{a}$ & 0,00 & 0,00 & 0,00 \\
\hline Servicios personales & 1137,82 & 1257,89 & 1497,94 \\
\hline Administración pública & 204,92 & 263,79 & 391,74 \\
\hline Total & 5181,02 & 5788,52 & 7102,14 \\
\hline
\end{tabular}

Fuente: Elaboración propia, sobre la base de datos del Instituto Nacional de Estadísticas y el Banco Central de Chile.

a El sector de servicios de vivienda no tiene trabajadores. En este sector se informan los gastos que se deberían pagar anualmente por usar las viviendas, por lo tanto, este sector no tiene empleados. 
El salario promedio se calcula como el cociente entre el total de pagos por concepto de salarios y el número de horas trabajadas:

$$
w=\frac{i^{\prime} l_{v}}{i^{\prime} l_{n}}
$$

donde $i$ es un vector unitario, $l_{v}$ es el vector de los pagos a los trabajadores en cada sector y $l_{n}$ es el vector que indica el número de horas trabajadas por sector.

El vector de coeficientes técnicos del trabajo, $l$, de cada año se calcula dividiendo el número de horas trabajadas en cada sector por el valor bruto de la producción sectorial, de la siguiente manera:

$$
l=\hat{x}^{-1} l_{n}
$$

donde, como se señaló anteriormente, $l_{n}$ es el vector de las horas trabajadas en cada sector y $x$ es el valor del producto bruto de cada sector, expresado en términos constantes.

\section{Precio del servicio del capital y los coeficientes técnicos del capital}

Para calcular los indicadores de capital, se utilizó información presentada en Estudios Económicos Estadísticos № 63 del Banco Central de Chile (Henríquez, 2008) y actualizada hasta el año 2010, conforme a los criterios establecidos en el Sistema de Cuentas Nacionales 2008 (Naciones Unidas, 2009) y ampliados en el manual sobre medición del capital de la OCDE (2009).

En lo que respecta a la información sobre el acervo de capital, hay importantes diferencias entre los datos necesarios y los disponibles (véase el cuadro 3).

Cuadro 3

Comparación de sectores considerados para el cálculo del capital

\begin{tabular}{ll}
\hline \multicolumn{1}{c}{ En este estudio } & \multicolumn{1}{c}{ En Henríquez (2008) } \\
\hline Agropecuario-silvícola & Agricultura, silvicultura y pesca \\
\hline Pesca & Minería \\
\hline Minería & Industria \\
\hline Industria manufacturera & Electricidad, gas y agua \\
\hline Electricidad, gas y agua & Construcción \\
\hline Construcción & Comercio, restaurantes y hoteles \\
\hline Comercio, hoteles y restaurantes & Transporte y comunicaciones \\
\hline Transporte y comunicaciones & Servicios financieros y empresariales \\
\hline Intermediación financiera y servicios empresariales & Servicios de vivienda \\
\hline Servicios de vivienda & Servicios comunales y sociales \\
\hline Servicios personales & \\
\hline Administración pública & \\
\hline
\end{tabular}

Fuente: Elaboración propia.

El capital del sector agricultura, silvicultura y pesca considerado por Henríquez (2008) es un dato agregado. Para distribuirlo entre los sectores agropecuario-silvícola y pesca, se supuso que la relación capital-producto es igual en ambos, por lo que el primero se calculó mediante las siguientes ecuaciones: 


$$
k_{1}=k_{\text {asp }} \frac{x_{1}}{x_{1}+x_{2}} \quad k_{2}=k_{\text {asp }} \frac{x_{2}}{x_{1}+x_{2}}
$$

donde $k_{a s p}$ es el acervo de capital del sector de agricultura, silvicultura y pesca, y $x_{1}$ y $x_{2}$ son el producto bruto de la agricultura y la silvicultura, por una parte, y la pesca, por otra. Finalmente, $k_{1}$ y $k_{2}$ son los valores del capital calculados para cada sector.

En el estudio de Henríquez (2008) no se proporciona información sobre el acervo de capital de la administración pública. Para determinar este valor en términos aproximados, se calculó la relación entre el capital y el producto de todos los sectores, excepto el de la administración pública.

$$
v=\frac{\sum_{i \neq g} k_{i}}{\sum_{i \neq g} x_{i}}
$$

A continuación, se supuso que la relación capital-producto de la administración pública es igual a la que presenta el sector de gobierno, lo que permitió calcularla a partir del producto del segundo.

$$
k_{g}=v \cdot x_{g}
$$

donde $x_{g}$ es la producción bruta consignada en las cuentas nacionales y $k_{g}$ es el acervo de capital estimado del gobierno.

El precio del servicio del capital se determinó de la siguiente manera:

$$
r=p_{k}\left(r_{0}+\delta_{k}-\frac{\dot{p}_{k}}{p_{k}}\right)
$$

Donde $r_{0}, \delta_{k}$ y $p_{k}$ representan la tasa de interés (costo de oportunidad), la tasa de depreciación y el precio (o plusvalía) del capital, respectivamente.

El valor agregado del capital, consignado por el Banco Central de Chile, puede definirse como se indica a continuación:

$$
k_{v}=r \phi k
$$

donde $r$ es el precio del servicio del capital y $\phi$ representa el factor de utilización, que indica la cantidad empleada (entre otros, por hora) del acervo de capital disponible, $k$, que es la expresión monetaria del acervo disponible con fines de producción (entre otros, equipos, automóviles y propiedades) adquirido en el pasado.

Para calcular el coeficiente de utilización del capital, es necesario emplear cantidades físicas, como se indica en la siguiente ecuación:

$$
c_{k}=\frac{\rho_{k} \phi k}{\rho_{x} x}
$$

donde $\rho$ es el deflactor que permite relacionar con un año base 0 las medidas de capital $k$ y el producto bruto $x$, y $\phi$ es la tasa de utilización del capital. Por lo tanto, el coeficiente $c_{k}$ (conocido como coeficiente técnico de producción) representa el capital físico necesario para producir una unidad de producto. Nótese que este concepto de físico tiene el sentido de un coeficiente técnico de producción.

Para calcular el precio del servicio del capital se emplea la siguiente ecuación: 


$$
r=\frac{k_{v}}{\phi K}
$$

En este caso, se entiende claramente que cuando se divide el resultado por la utilización efectiva del capital, $\phi K$, se puede determinar el precio, ya que $r=\frac{k_{v}}{\phi K}=\frac{r \phi K}{\phi K}=\frac{r \phi}{\phi}$.

\section{Productividad total de los factores}

Mediante la aplicación del procedimiento de reglas de cálculos escrito en el anexo, se calcularon, a partir de las matrices de uso y producción, las matrices de coeficientes técnicos necesarias para determinar el cambio en la PTF entre los tres períodos considerados, conforme a la ecuación (4).

Los resultados de este cálculo se sintetizan en el cuadro 4, en el que también se presentan las tasas de incremento del valor agregado.

\section{Cuadro 4}

PTF agregada y participación en el crecimiento

(En porcentajes)

\begin{tabular}{lccc}
\hline & $\begin{array}{c}\text { Crecimiento del valor agregado } \\
(1)\end{array}$ & $\begin{array}{c}\text { Crecimiento de la PTF } \\
\text { (2) }\end{array}$ & $\begin{array}{c}\text { Participación de la PTF } \\
\text { (2)/(1) }\end{array}$ \\
\hline $1996-2003$ & 27,15 & 4,95 & 18,23 \\
\hline $1996-2003$ (anual) & 3,491 & 0,693 & 15,66 \\
\hline $2003-2010$ & 36,07 & 5,65 & \\
\hline $2003-2010$ (anual) & 4,498 & 0,788 & \\
\hline
\end{tabular}

Fuente: Elaboración propia, sobre la base de datos del Instituto Nacional de Estadísticas y el Banco Central de Chile. Nota: PTF: Productividad total de los factores.

Si bien la PTF de 1996-2003 fue inferior a la de 2003-2010, su participación como fuente de crecimiento fue superior en el primer período, lo que se observa en la última columna del cuadro.

En el cuadro 5 se presenta el resultado de los cálculos de la PTF de Chile consignados en otros estudios referidos a los mismos períodos. Cabe destacar la notable heterogeneidad de los resultados, que obedece al empleo de diferentes fuentes de datos y a la aplicación de distintos métodos. 
Cuadro 5

PTF anual calculada en distintos estudios

(En porcentajes)

\begin{tabular}{lc}
\hline Período & PTF anual \\
\hline $1998-2005^{a}$ & 1,89 \\
\hline $1990-2005^{a}$ & 3,06 \\
\hline $1996-2003^{\text {a }}$ & 0,6 \\
\hline $2003-2005^{\text {b }}$ & 1,74 \\
\hline $1996-2000^{c}$ & 0,2 \\
\hline $2000-2003^{c}$ & $-0,1$ \\
\hline $1998-2005^{d}$ & 1,77 \\
\hline $1998-2005^{a}$ & 0,66 \\
\hline $2000-2008^{f}$ & 1 \\
\hline
\end{tabular}

Fuente: Elaboración propia, sobre la base de R. Fuentes, M. Larraín y K. Schmidt-Hebbel, "Sources of growth and behavior of TFP in Chile", Cuadernos de Economía, vol. 43, № 127, Santiago, Pontificia Universidad Católica de Chile, 2006; R. Vergara y R. Rivero, "Productividad sectorial en Chile 1986-2001", Cuadernos de Economía, vol. 43, № 127, Santiago, Pontificia Universidad Católica de Chile, 2006; R. Fuentes y M. Morales, "On the measurement of total factor productivity: a latent variable approach", Macroeconomic Dynamics, vol. 15, № 2, Cambridge University Press, 2011; e I. Magendzo, Evolución de la productividad total de los factores, Santiago, Universidad Adolfo lbáñez, 2013.

Nota: PTF: Productividad total de los factores.

a Fuentes, Larraín y Schmidt-Hebbel (2006).

b Los autores no presentan información sobre el crecimiento de la PTF en estos períodos, sino índices de la PTF anual, que sirvieron de base para el cálculo de los datos consignados en el cuadro. En particular, se utilizó el índice de PTF donde se corrigió el capital y el empleo por horas de trabajo y salarios, respectivamente.

c Vergara y Rivero (2006).

d Fuentes y Morales (2011), sobre la base de contabilidad del crecimiento.

e Ibíd., sobre la base de espacios de estados.

f Magendzo (2013).

Las cifras correspondientes a 1996-2003 presentadas en el cuadro 4 son similares a los resultados del cálculo de la PTF de esos mismos años expuestos por Fuentes, Larraín y SchmidtHebbel (2006). Esta similitud se da en dos dimensiones, dado que el crecimiento anual de la PTF en este período fue de un 0,6\%, mientras que la participación de esta en el crecimiento de la economía fue de un $18,92 \%$.

Para comprender la fuente del cambio en la PTF, en el cuadro 6 se presenta la descomposición realizada en la ecuación (5). En la primera línea de la primera columna se indica la diferencia entre la productividad de los períodos 1996-2003 y 2003-2010. El valor se descompone conforme a las cifras que figuran en las siguientes tres columnas. En la segunda fila se indica la participación de cada componente en el cambio. La mayor parte del que se produjo entre los dos períodos considerados se debe a la variación de la productividad intrasectorial e intersectorial, cuyo total corresponde a un efecto de aproximadamente el $80 \%$ del cambio.

Cuadro 6

Descomposición de la PTF

\begin{tabular}{lcccc}
\hline & $\begin{array}{c}\text { Cambios en la demanda } \\
\text { sectorial (1) } \\
\pi S \Delta \beta\end{array}$ & $\begin{array}{c}\text { Cambios en la interacción } \\
\text { entre sectores (2) } \\
\pi S \Delta \beta\end{array}$ & $\begin{array}{c}\text { Cambios en la PTF } \\
\text { de cada sector (3) } \\
\pi S \Delta \beta\end{array}$ & $\begin{array}{c}\text { Total } \\
(1)+(2)+(3)\end{array}$ \\
\hline$\Delta \rho=0,065-0,0495$ & 0,001344 & 0,003134 & 0,002474 & 0,006949 \\
\hline Porcentaje & $19,34 \%$ & $45,1 \%$ & $35,6 \%$ & $100,06 \%^{\mathrm{a}}$
\end{tabular}

Fuente: Elaboración propia.

Nota: PTF: Productividad total de los factores.

a El valor supera el 100\%, debido a errores de aproximación de segundo orden. 
En el cuadro 7 se presenta la descomposición del 35,6\% del cambio observado de la PTF de toda la economía. En la segunda columna se indican los valores de $w=S \beta$, que es un vector de 12 sectores. Dado que el vector $\beta$ puede interpretarse como la distribución del gasto de todos los sectores de la economía expresado en una unidad monetaria, la posición $i$ del vector $w(i)$ representa el aumento de la actividad económica del sector $i$, medida en términos del precio del bien que este produce cuando se incrementa la demanda agregada de la economía en una unidad. Lo anterior significa que el vector puede interpretarse como una medida de los encadenamientos hacia atrás de cada sector debidos al aumento de la demanda agregada de la economía en una unidad. Estos encadenamientos pueden incrementarse, ya sea porque el precio del bien de un sector aumenta en relación con el precio de los bienes de todos los demás o porque efectivamente se da un cambio tecnológico que eleva el multiplicador técnico sectorial. En el cuadro 7 la industria manufacturera aparece como el sector que muestra mayores encadenamientos hacia atrás medidos en términos monetarios, seguido por el sector de comercio, hoteles y restaurantes. En la tercera columna se indica el cambio tecnológico de cada sector, lo que permite concluir que el de electricidad, gas y agua fue el que registró un mayor aumento de la productividad, seguido por los de pesca, y de intermediación financiera y servicios empresariales.

Por último, en la cuarta columna se presenta el resultado de la combinación de los efectos. Este revela que cuatro sectores contribuyeron positivamente y en medidas similares al cambio: electricidad, gas y agua; intermediación financiera y servicios empresariales; servicios de vivienda, e industria manufacturera. Los sectores de servicios personales y construcción son los que hicieron una contribución más negativa al cambio tecnológico.

Cuadro 7

Análisis del 35,6\% del cambio

\begin{tabular}{lccc}
\hline Sector & $\begin{array}{c}w=S \beta \\
(1)\end{array}$ & $\begin{array}{c}\Delta \pi \\
(2)\end{array}$ & $(1)^{\star}(2)$ \\
\hline Agropecuario-silvícola & 0,1042 & $-0,0033$ & $-0,00034$ \\
\hline Pesca & 0,0196 & 0,0373 & 0,00073 \\
\hline Minería & 0,1218 & 0,0075 & 0,00091 \\
\hline Industria manufacturera & 0,3466 & 0,0066 & 0,00229 \\
\hline Electricidad, gas y agua & 0,0828 & 0,04 & 0,00331 \\
\hline Construcción & 0,1078 & $-0,0264$ & $-0,00285$ \\
\hline Comercio, hoteles y restaurantes & 0,2138 & 0,0009 & 0,00019 \\
\hline Transporte y comunicaciones & 0,1406 & $-0,009$ & $-0,00127$ \\
\hline Intermediación financiera y servicios empresariales & 0,1146 & 0,0278 & 0,00319 \\
\hline Servicios de vivienda & 0,2003 & 0,0149 & 0,00298 \\
\hline Servicios personales & 0,2096 & $-0,0255$ & $-0,00534$ \\
\hline Administración pública & 0,0618 & $-0,0216$ & $-0,00133$ \\
\hline Efecto total debido a los cambios en el PTF intrasectorial & & & 0,00247 \\
\hline
\end{tabular}

Fuente: Elaboración propia.

Nota: PTF: Productividad total de los factores.

El otro componente importante del cambio de la PTF se analiza en el cuadro 8. Empleando el mismo método utilizado en el cálculo anterior, se analizó la composición del término $\pi \Delta S \beta$, desagregado en dos partes. En primer lugar, se analizó el vector intermedio $z=\pi \Delta S$ de dimensión (12x1), compuesto por el vector de la PTF sectorial, $\pi$, y la matriz de cambios en los encadenamientos, $\Delta S$. Cada elemento $\Delta S_{i, j}$ representa el efecto del cambio en los encadenamientos hacia atrás del sector $j$, medidos en términos monetarios, en el sector $i$, como consecuencia de la producción de una unidad adicional de $j$ para satisfacer la demanda. Si $\Delta S_{i, j}<0,\left(\Delta S_{i, j}>0\right)$, en el nuevo período el sector $j$ 
sería menos (o más) importante para el sector $i$. En la columna $\Delta S_{\text {.j }}$ se indica el cambio producido por el sector $j$ en todo el tejido productivo, medido con los multiplicadores monetarios. Esta información permite interpretar $z(i)$ como la difusión de los cambios tecnológicos en todos los sectores, y por lo tanto en el sector $i$, como consecuencia de las variaciones de los multiplicadores. Cuanto mayor sea $z(i)$, más importante será el efecto tecnológico que difunda el sector $i$.

Cuadro 8

Análisis del 45,1\% del cambio en la PTF

\begin{tabular}{lccc}
\hline Sector & $z=\pi \Delta S$ & $\begin{array}{c}B \\
(1)\end{array}$ & $(1)^{\star}(2)$ \\
\hline Agropecuario-silvícola & 0,0059 & 0,0351 & 0,00021 \\
\hline Pesca & 0,0209 & 0,0033 & 0,00007 \\
\hline Minería & 0,0044 & 0,0824 & 0,00036 \\
\hline Industria manufacturera & 0,0099 & 0,0963 & 0,00095 \\
\hline Electricidad, gas y agua & 0,0278 & 0,0179 & 0,00050 \\
\hline Construcción & $-0,0008$ & 0,0933 & $-0,00007$ \\
\hline Comercio, hoteles y restaurantes & $-0,0044$ & 0,1376 & $-0,00061$ \\
\hline Transporte y comunicaciones & 0,0031 & 0,0603 & 0,00019 \\
\hline Intermediación financiera y servicios empresariales & 0,006 & 0,027 & 0,00016 \\
\hline Servicios de vivienda & 0,0037 & 0,2003 & 0,00074 \\
\hline Servicios personales & 0,0041 & 0,1872 & 0,00077 \\
\hline Administración pública & $-0,0021$ & 0,0592 & $-0,00012$ \\
\hline Efecto total debido a los efectos intersectoriales en el cambio de la PTF & & & 0,00314 \\
\hline
\end{tabular}

Fuente: Elaboración propia.

Nota: PTF: Productividad total de los factores.

La cuarta columna del cuadro 8 puede interpretarse como el avance tecnológico difundido en todo el sistema productivo, siempre que la tecnología y las preferencias de los consumidores se mantengan constantes.

El sector de industria manufacturera es el que más difunde los avances tecnológicos en todo el sistema productivo, seguido de los servicios de vivienda y los servicios personales. Al parecer, los sectores de construcción, administración pública y comercio, hoteles y restaurantes registraron una reducción de su influencia en el tejido productivo, por lo que también disminuyeron los efectos de su interacción con los demás.

En el diagrama 1 se presenta una síntesis de los efectos de los dos componentes más importantes de la descomposición. En el eje horizontal se muestran los efectos intrasectoriales de cada sector en la PTF, mientras que en el eje vertical se indican los efectos de difusión intersectoriales. A partir de estas dos dimensiones, se distinguen cuatro categorías: en el cuadrante I se agrupan los sectores en los que tanto la PTF como los efectos de los encadenamientos tecnológicos con otros se incrementaron. En estos sectores no solo se observa un perfeccionamiento de las tecnologías empleadas, sino que también se constata que las difundieron a toda la economía. El cuadrante II está compuesto por sectores cuya PTF disminuyó, pero en los que aumentó la intensidad de los encadenamientos intersectoriales. Por este motivo, es posible que actúen como factores limitantes del crecimiento tecnológico, ya que su baja productividad se difunde a todo el sistema productivo. El cuadrante III está integrado por sectores con una PTF negativa, pero cuyos efectos en toda la economía se redujeron, por lo que no limitan la expansión de la productividad. Finalmente, en el cuadrante IV se incluyen los sectores en los que aumentó la productividad sectorial, pero cuyos efectos en el resto de la economía se redujeron, por lo que deberían integrarse más al sistema económico. 


\section{Diagrama 1}

PTF: efectos intersectoriales e intrasectoriales

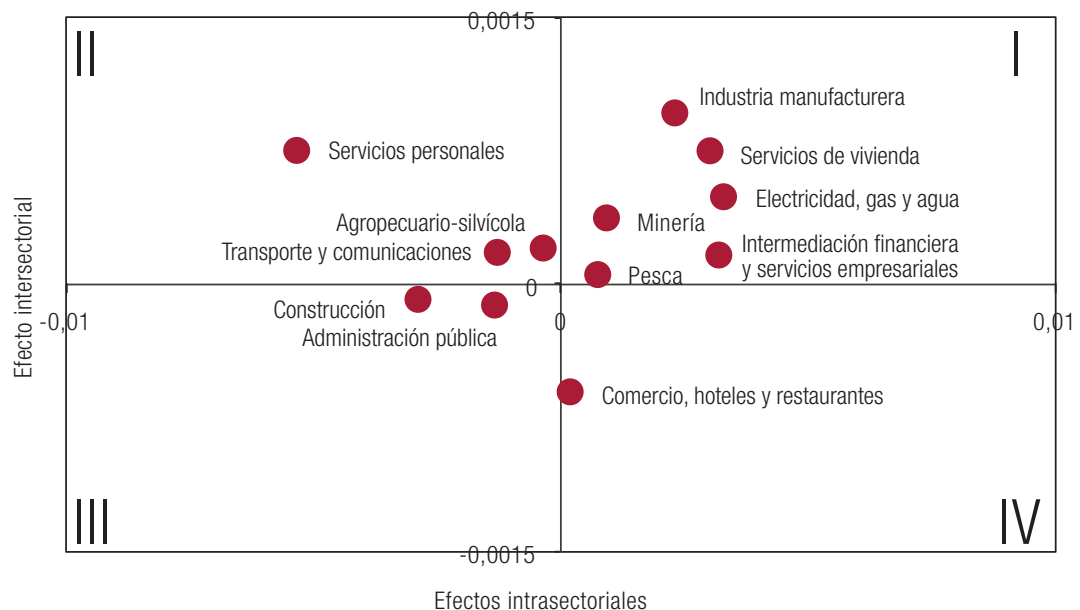

Fuente: Elaboración propia.

Nota: PTF: Productividad total de los factores.

Cuatro de los sectores incluidos en el cuadrante I destacan por el aumento de la productividad y sus efectos en el sistema productivo: industria manufacturera; servicios de vivienda ${ }^{8}$; electricidad, gas y agua, e intermediación financiera y servicios empresariales. Por sus menores efectos, les sigue el sector de minería.

En el cuadrante II se observa un predominio del sector de servicios personales. Los más importantes de esta categoría son la educación, la salud, las actividades de servicios sociales y asociaciones, las actividades de esparcimiento, la gestión de desechos y una variada gama de servicios, entre otros el lavado y la limpieza de prendas de tela y piel, los servicios de las peluquerías, los servicios fúnebres, el servicio doméstico y otros. Se trata principalmente de servicios prestados a los hogares.

En el cuadrante III se incluyen dos sectores: construcción y administración pública. El cambio en el efecto del primero de estos en el tejido productivo es bajo y, además, cabe destacar que registra una considerable reducción de la PTF.

El cuadrante IV está integrado por el sector de comercio, hoteles y restaurantes, que muestra un muy escaso aumento de la productividad y, a la vez, una notable disminución de su efecto en la economía.

\section{Conclusiones}

Las conclusiones del presente estudio invitan a considerar la intensidad del cambio en la productividad y a realizar un análisis hipotético de sus causas.

La intensidad del cambio se refleja en el resultado del cálculo realizado a partir de la ecuación (4):

$$
\Delta \rho=\rho_{03,10}-\rho_{96,03}=0,0565-0,0495=0,007=0,7 \%
$$

\footnotetext{
8 De acuerdo con la definición del Banco Central de Chile, el sector de servicios de vivienda comprende los servicios de las viviendas utilizadas con fines habitacionales. En la práctica, en la cuenta de producción de esta actividad se consigna el valor de los arriendos pagados por los arrendatarios y el valor imputado de las viviendas habitadas por sus propietarios. Este sector tiene una importante participación en la demanda final. Todo aumento de su productividad obedece fundamentalmente al incremento del valor agregado, que se calcula como una proporción del acervo neto valorizado de viviendas. Por lo tanto, convendría considerar con cautela la información proporcionada por este sector para determinar el efecto que ejerce en la productividad.
} 
donde $\rho_{03,10}$ y $\rho_{96,03}$ representan la PTF calculada para los períodos 2003-2010 y 1996-2003, respectivamente. El resultado indica que el aumento de la productividad entre los dos períodos fue de un $0,7 \%$. Cabe destacar que esta acentuación de la eficiencia se produjo en el período 20032010, en el que la expansión del PIB fue superior a la registrada en 1996-2003 (véase el cuadro 4). Por consiguiente, en términos relativos en el segundo período la participación del incremento de la eficiencia fue inferior a la del primero. Estas conclusiones confirman la advertencia sobre el escaso crecimiento de la economía atribuible al factor "inspiración", formulada en el análisis de la contabilidad del crecimiento de Chile (véase Fuentes, Larraín y Schmidt-Hebbel, 2006).

Existen dos hipótesis, no necesariamente excluyentes, sobre los cambios de la PTF. Según la primera de ellas y dado el carácter procíclico que Fuentes, Larraín y Schmidt-Hebbel (2006) atribuyen a la PTF, los años seleccionados para la elaboración de las matrices de insumo-producto empleadas en este estudio y las características del ciclo económico de Chile (véase el gráfico 1), es posible que el aumento absoluto de la PTF haya obedecido a factores cíclicos como la mejora de los términos de intercambio, inducida por el aumento del precio del cobre en el período analizado (véase Fuentes, Larraín y Schmidt-Hebbel, 2006). Esta primera hipótesis, de orden macroeconómico, se complementa con la segunda, de orden microeconómico, referida a un canal de transmisión específico que puede haber contribuido a sostener el nivel de la PTF.

La segunda hipótesis es producto del cruce de la descomposición por sectores realizada en este estudio y sintetizada en el diagrama 1, por una parte, y los resultados expuestos por Fernandes y Paunov (2012), por otra. En la síntesis presentada en dicho diagrama se observa la influencia positiva ejercida en el cambio de la productividad por los sectores manufacturero; de electricidad, gas y agua, y de intermediación financiera y servicios empresariales. En cambio, en el estudio de Fernandes y Paunov se vinculan estos tres sectores en función del efecto del considerable incremento de la inversión extranjera directa (IED) en el sector servicios en el período estudiado. En la década de 1990, la IED en el sector servicios representó aproximadamente el 60\% de esta inversión en Chile, lo que, en virtud de mecanismos de reducción de precios, mejoras de calidad, acentuación de la diversificación y difusión de conocimientos, influyó positivamente en la PTF de las empresas que integran el sector manufacturero.

El presente estudio tenía por objeto estudiar la composición sectorial de los cambios en la PTF registrados en Chile desde 1996 hasta 2010, en el marco de la contabilidad del crecimiento de Solow y tomando en consideración la interacción intrasectorial. Para realizar este ejercicio, se empleó la información de las matrices de insumo-producto de los años 1996, 2003 y 2010 proporcionadas por el Banco Central de Chile. En la parte II se describe el método de descomposición empleado, mientras que en la III y en el anexo se exponen los cálculos realizados para obtener la información básica, y en la IV se analizan los resultados obtenidos.

La descomposición de la PTF se realizó en tres etapas referidas, respectivamente, a la productividad intrasectorial, sus efectos de difusión y los cambios en la demanda que influyen en la productividad. Se determinó que más del $80 \%$ del cambio en la productividad en el período analizado obedeció a los dos primeros factores.

Los resultados de esta investigación permiten construir una taxonomía de sectores, en función de su capacidad para dar impulso a la economía y los cambios en la productividad sectorial. En esta taxonomía destacan la industria manufacturera y los sectores de electricidad, gas y agua, e intermediación financiera y servicios empresariales, que encabezaron el aumento de la PTF en el período 1996-2010. El incremento de la productividad de estos sectores se difundió a todo el tejido productivo.

Por registrar un descenso de la productividad durante el período, que ejerció una gran influencia en el sistema productivo, el sector de servicios personales podría haber limitado el crecimiento de la productividad de toda la economía. 


\section{Bibliografía}

Álvarez, R. y R. Fuentes (2009), "Labor market regulations and productivity: evidence from Chilean manufacturing plants”, Documento de Trabajo, № 396, Santiago, Pontificia Universidad Católica de Chile.

(2004), "Patrones de especialización y crecimiento sectorial en Chile", Documentos de Trabajo, № 288, Santiago, Banco Central de Chile.

Baumol, W.J. y E.N. Wolff (1984), "On interindustry differences in absolute productivity", Journal of Political Economy, vol. 92, № 6, Chicago, The University of Chicago Press.

Bergoeing, R., A. Hernando y A. Repetto (2005), "Market reforms and efficiency gains in Chile", Documentos de Trabajo, № 372, Santiago, Banco Central de Chile.

Chumacero, R.A. y R. Fuentes (2006), "Chilean growth dynamics", Economic Modelling, vol. 23, № 2, Amsterdam, Elsevier.

Del Gatto, M., A. Di Liberto y C. Petraglia (2011), "Measuring productivity", Journal of Economic Surveys, vol. 25, No 5 , Wiley.

Easterly, W. y R. Levine (2002), "It's not factor accumulation: stylized facts and growth models", Economic Growth: Sources, Trends, and Cycles, N. Loayza, R. Soto y K. Schmidt-Hebbel (eds.), Santiago, Banco Central de Chile.

Fernandes, A.M. y C. Paunov (2012), "Foreign direct investment in services and manufacturing productivity: evidence for Chile", Journal of Development Economics, vol. 97, № 2, Amsterdam, Elsevier.

Fuentes, R. (2011), "Una mirada desagregada del deterioro de la productividad en Chile: ¿existe un cambio estructural?, Documento de Trabajo, № 401, Santiago, Pontificia Universidad Católica de Chile.

Fuentes, R. y M. Morales (2011), "On the measurement of total factor productivity: a latent variable approach", Macroeconomic Dynamics, vol. 15, № 2, Cambridge University Press.

Fuentes, R., M. Larraín y K. Schmidt-Hebbel (2006), "Sources of growth and behavior of TFP in Chile", Cuadernos de Economía, vol. 43, № 127, Santiago, Pontificia Universidad Católica de Chile.

Harberger, A.C. (1998), "A vision of the growth process", American Economic Review, vol. 88, № 1, Nashville, Tennessee, American Economic Association.

Henríquez, C. (2008), "Stock de capital en Chile (1985-2005): metodología y resultados", Estudios Económicos Estadísticos, № 63, Santiago, Banco Central de Chile.

Hodrick, R.J. y E.C. Prescott (1981), "Postwar U.S. business cycles: an empirical investigation", Discussion Papers, N ${ }^{\circ} 451$, Northwestern University.

Magendzo, I. (2013), Evolución de la productividad total de los factores, Santiago, Universidad Adolfo lbáñez.

Miller, R. y P.D. Blair (2009), Input-output Analysis: Foundations and Extensions, Nueva York, Cambridge University Press.

OCDE (Organización para la Cooperación y el Desarrollo Económicos) (2009), Measuring Capital. OECD Manual, París.

(2001), Measuring Productivity. Measurement of Aggregate and Industry-level Productivity Growth, París.

Naciones Unidas (2009), Sistema de Cuentas Nacionales 2008, Nueva York, Comisión Europea/Fondo Monetario Internacional/Organización para la Cooperación y el Desarrollo Económicos/Naciones Unidas.

Pavcnik, N. (2002), "Trade liberalization, exit, and productivity improvements: evidence from Chilean plants", Review of Economic Studies, vol. 69, № 1, Oxford University Press.

Prescott, C.E. (1998), "Needed: a theory of total factor productivity", International Economic Review, vol. 39, $N^{\circ} 3$.

Solow, R.M. (1957), "Technical change and the aggregate production function", Review of Economics and Statistics, vol. 39, № 3.

Timmer, M.P. y otros (2010), Economic Growth in Europe. A Comparative Industry Perspective, Cambridge University Press.

Vergara, R. y R. Rivero (2006), "Productividad sectorial en Chile 1986-2001", Cuadernos de Economía, vol. 43, № 127, Santiago, Pontificia Universidad Católica de Chile.

Wolff, E.N. (1985), "Industrial composition, interindustry effects, and the U.S. productivity slowdown", The Review of Economics and Statistics, vol. 67, Nㅜㄹ, Cambridge, Massachusetts, The MIT Press.

(1984), "Industrial composition, interindustry effects, and the U.S. productivity slowdown", Economic Research Reports, № 84-09, Nueva York, Universidad de Nueva York. 


\section{Anexo A1}

\section{Datos y métodos de cálculo empleados}

En este anexo se describen los cálculos realizados conforme a los tres métodos indicados.

\section{Cálculo de los coeficientes técnicos}

Los datos proporcionados por el Banco Central de Chile se agrupan en matrices de producción y uso (véase Miller y Blair, 2009), que fueron la principal fuente considerada para el cálculo de las matrices de transacciones intersectoriales.

Se empleó el modelo de Miller y Blair (2009) basado en la demanda, conforme al cual la tecnología está determinada por la estructura de producción de bienes, independientemente de la industria de los que provengan, para desarrollar las matrices de requerimientos totales. En términos analíticos,

$U$ : matriz de usos con 13 productos por 12 sectores.

$V$ : matriz de producción sectorial con 12 sectores por 13 productos.

$s=V^{*} i$ : vector de producción industrial o sectorial $(12 \times 1)$.

$Q=V * i$ : vector de producción por commodities $(13 \times 1)$.

Para simplificar, no se tomaron en cuenta los subíndices temporales.

Sobre la base de esta información, se calculó la matriz de transacciones intersectoriales de la siguiente manera:

$$
\begin{aligned}
& B=U \cdot \hat{s}^{-1} \\
& D=V \cdot \hat{q}^{-1} \\
& Z=D \cdot B \cdot \hat{s}
\end{aligned}
$$

En este caso, se coloca el signo de acento circunflejo sobre los vectores (por ejemplo, $\hat{s}$ ) para indicar su diagonalización. En el cálculo de la matriz de coeficientes $A$, se sumaron las matrices de compras nacionales y de importaciones. Por lo tanto, a la matriz de transacciones calculada se le agrega la siguiente información, también provista por el Banco Central de Chile:

M: matriz de importaciones por cada sector de $12 \times 12$.

$L_{v}$ : vector fila con el Valor Agregado del Salariopor sector de 12x1.

$K_{v}$ : vector fila con el Valor agregado del Capital por sector de $12 \times 1$.

$t_{v}$ : vector fila de impuestos indirectos de $12 \times 1$.

El vector columna de la producción total se calcula como se indica a continuación:

$$
x=i^{\prime} Z+i^{\prime} M+l_{v}+k_{v}+t_{v}
$$

Para calcular el vector columna de la demanda final, se identifican los productos de cada sector y se les restan las correspondientes ventas intermedias.

$$
y=x-i^{\prime} Z^{\prime}-i^{\prime} M^{\prime}
$$




\section{Cálculo del vector de productividad sectorial}

Dado que la productividad se calcula para distintos períodos, se emplea la aproximación propuesta por Wolff (1984):

$$
\begin{aligned}
& \beta_{i}=\frac{y_{i}}{Y} \\
& v_{L}=\frac{w L}{Y} \\
& v_{k}=\frac{r K}{Y}
\end{aligned}
$$

Los valores promedio de dos períodos se representan de la siguiente manera:

$$
\bar{\beta}_{i}=\frac{\beta_{i, t}+\beta_{i, t+1}}{2}
$$

El valor aproximado de la PTF agregada se puede calcular mediante la siguiente ecuación:

$$
\rho \cong \sum_{j} \bar{\beta}_{j} \Delta \log y_{j}-\bar{v}_{L} \Delta \log L-\bar{v}_{K} \Delta \log K
$$

El valor aproximado de la PTF del sector $j$ se calcula de manera similar:

$$
\pi_{j} \cong-\left(\sum_{i} \bar{\alpha}_{i, j}\left(\Delta \log a_{i, j}\right)-\bar{v}_{L, j}\left(\Delta \log l_{j}\right)-\bar{v}_{K, j}\left(\Delta \log k_{j}\right)\right)
$$

donde $\alpha_{i, j}=\frac{p_{i} a_{i, j}}{p_{j}}, v_{L, j}=\frac{w l_{j}}{p_{j}}, v_{K, j}=\frac{r k_{j}}{p_{j}}$. El guion agregado encima de las variables indica que se utilizaron los ponderadores del índice de Turnquist-Divisia para varios períodos. 